\title{
Anabases
}

ANABASES Traditions et réceptions de l'Antiquité

22 | 2015

Varia

\section{Entre graffiti et graff : un simple écart de langage ? À propos de deux livres récents}

\section{Manuel Royo}

\section{(2) OpenEdition}

1 Journals

Édition électronique

URL : http://journals.openedition.org/anabases/5507

DOI : 10.4000/anabases.5507

ISSN : 2256-9421

Éditeur

E.R.A.S.M.E.

\section{Édition imprimée}

Date de publication : 20 octobre 2015

Pagination : 253-257

ISSN : 1774-4296

\section{Référence électronique}

Manuel Royo, «Entre graffiti et graff : un simple écart de langage ? À propos de deux livres récents », Anabases [En ligne], 22 | 2015, mis en ligne le 20 octobre 2018, consulté le 21 octobre 2019. URL: http://journals.openedition.org/anabases/5507 ; DOI : 10.4000/anabases.5507 
Labex ARCHIMEDE (programme ANR-11-LABX-0032-01) helene.menard@univ-montp3.fr

\section{Les mots de l’Antiquité}

\section{Entre graffiti et graff: un simple écart de langage? À propos de deux livres récents ${ }^{64}$}

\section{Manuel Royo}

En 2012 s'est tenue à Nîmes, dans la chapelle des Jésuites, une exposition sur les graffitis gallo-romains. Réalisée par Alix Barbet et Michel Fuchs, elle avait été initialement présentée au Musée romain de Lausanne-Vidy. Son titre, Les Murs murmurent, fait explicitement référence au documentaire d'Agnès Varda tourné en I98I sur les murs peints et les graffitis de Los Angeles ${ }^{65}$. La cinéaste, sous le charme de ces murs, partait à la rencontre des artistes et des communautés, multipliant les éclairages (ethnologique, sociologique, historique, politique ou plastique) d'un “art» éminemment éphémère.

La référence ne doit pas étonner. Telle est un peu la marque du directeur du Musée romain de Lausanne, Laurent Flutsch, qui est bien connu pour avoir conçu ces dernières années d'autres expositions où il proposait de lire l'Antiquité à l'aune des pratiques du présent. Quand il ne relie pas l'antiquité lémanique à notre quotidien ${ }^{66}$ afin de la rendre présente et vivante, il imagine de nous projeter dans l'avenir burlesque d'une exposition des “trésors» de notre ordinaire contemporain, revus et réinterprétés, évidemment de façon fantaisiste, par des archéologues du $\mathrm{V}^{\mathrm{e}}$ millénaire: mise en perspective des lacunes de notre savoir sur le passé, du grossissement des effets de la mémoire, mais aussi leçon d'humilité vis-à-vis de notre ignorance ${ }^{67}$.

64 A. BaRbet et M. Fuchs (dir.), Les Murs murmurent. Graffitis gallo-romains, Musée romain de Lausanne-Vidy, Gollion, Infolio éditions, 20I2; Ch. Guichard, Graffitis. Inscrire son nom à Rome, $X V I^{e}-X I X^{e}$ siècle, Paris, Le Seuil, 20I4.

65 Mur Murs, documentaire d'A. VARDA, 1982.

66 PasséPrésent. Lousonna ou l'Antiquité d'actualité, catalogue de l'exposition permanente “Lousonna Passé Présent », Musée romain de Lausanne-Vidy, Gollion, Infolio éditions, 2004 .

67 Futur antérieur. Trésors archéologiques du $2 I^{e}$ siècle après J.-C., Musée romain de Lausanne-Vidy, Gollion, Infolio éditions, 2002. 
C'est une passerelle en sens inverse, comme dans Passé Présent, qu'il suggère de tendre entre graffs contemporains et graffitis antiques au travers de la présentation d'une centaine d'exemplaires gallo-romains rassemblés thématiquement et commentés par les auteurs du catalogue. Référence est explicitement faite, dès le préambule, aux tags et graffs contemporains, à cette “littérature de pissotière », exemples à l'appui, qui apostrophent comme peuvent le faire à deux mille ans de distance les témoignages de leurs lointains auteurs.

De manière tout aussi symbolique le catalogue s'ouvre sur l'image d'un mur peint comme une fresque antique où le visiteur de l'exposition était invité à laisser la trace de son passage par un graffiti et se conclut sur celle d'une fresque expérimentale du CEPMR ${ }^{68}$ sur laquelle la commissaire de l'exposition, Alix Barbet, a gravé, respectant les vides d'une paroi volontairement lacunaire, un SIC TRA[nsit g]LORIA [mu]NDI. Fragments de paroi et de lettres mettent ainsi en résonance le caractère doublement éphémère de ces vestiges comme du geste dont ils conservent trace, tandis qu'en écho la formule chrétienne vient pasticher le prétendu fac-similé de l’Antique.

En ce domaine et compte tenu des rapprochements que fait Laurent Flutsch dès le préambule du catalogue, peut-on considérer ces graffitis antiques au prisme de nos catégories contemporaines? À rebours, dans quelle mesure graffs et tags actuels s'inscriraient-ils dans une certaine forme de réception d'une pratique antique qu'ils renouvelleraient alors? Écriture d'impulsion, officieuse et ancrée dans la vie quotidienne, le graffiti antique se prête volontiers à une approche historienne en donnant à celle-ci l'épaisseur humaine que textes épigraphiques officiels, écrits littéraires ou vestiges monumentaux ont parfois du mal à animer. Henri Labrouste, architecte Prix de Rome, ajouta ainsi des graffitis sur les murs et les colonnes de sa restauration du Temple de Neptune de Pæstum soumise à l'Académie en I829 ${ }^{69}$. À leur tour, deux ouvrages récemment parus sont revenus sur la «matérialité » et les rapports avec la littérature de cette écriture si différente ${ }^{70}$, parole “écrite» qui dit l'instant d'une société ${ }^{71}$ lors même que son corpus, sa langue et parfois sa signification paraissent excéder notre capacité à en tracer les limites. Le choix des rubriques du catalogue (“comptes, poids et dates », " exercices de stylet », " rois de l'arène», “chasse et nature», etc.) traduit le désir de réduire l'altérité de ces graffitis que souligne encore leur difficile lisibilité et d'affirmer qu'au-delà des contingences historiques de nos sociétés respectives, les graffitis relèvent d'une commune humanité, travaillée par les mêmes préoccupations que celle-ci continue d'afficher depuis toujours sur ses murs : identité, sexe, pouvoir, argent, jeu, etc. Le parallèle est effectivement tentant entre ces sexes gravés sur le portique des villas d'Yvonand ou de Saint-Pierre-de-Nazac et

68 Centre d'études des peintures murales romaines, Abbaye de Saint-Jean-des-Vignes, Soissons, in Les Murs murmurent, p. I93, n 88, fig. I64.

69 Cité par Guichard, Graffitis, p.35-37.

70 J. A. Baird et C. TaYlor (dir.), Ancient Graffiti in Context, New York, Routledge, 20II; K. Milnor. Graffiti and the Literary Landscape in Roman Pompeii, Oxford, Oxford University Press, 2014.

71 Les Murs murmurent, p. 165 sq. 
ceux dessinés à la craie sur tel portone de Rome aujourd'hui, ou entre ces noms inscrits, comme au hasard, sur des parois antiques et ceux, tracés à la bombe, sur les murs ou le mobilier de nos villes.

Il n'est cependant pas sûr qu'à faire abstraction des contextes, nous puissions aller au-delà de ces idées générales. À bien y regarder, le plus petit commun dénominateur à ces pratiques éloignées dans le temps se résume au désir de laisser la trace passagère d'un geste lui-même éphémère.

Lorsqu'Alix Barbet réalise un pastiche de graffiti antique, elle reprend sciemment les codes qui accompagnaient la pratique qu'elle imite. Même si elle est moins explicite, l'invitation faite aux visiteurs de graver une trace de leur passage sur un mur ad hoc prétend retrouver l'impertinence antique et joue avec humour sur la réception. Il s'agit bien de s'inspirer de l'Antique, d'en rejouer les pratiques et d'en exploiter les codes. Il en va exactement de même avec Les Tablettes de buis d'Apronenia Avitia de Pascal Quignard et la Venere degli Stracci de Michel Angelo Pistoletto, mimétisme formel pour le premier, détournement par l'arte povera, pour le second, des codes hérités du néo-classicisme qui remet en question notre monde consumériste. Mutatis mutandis, graffeurs et tagueurs seraient-ils sans le savoir les héritiers de leurs ancêtres gallo-romains? Le marquage symbolique auquel ils se livrent dans l'espace urbain s'adresse en fait à un autre monde. Si les contenus peuvent être parfois identiques - revendications, provocations, apostrophes comme sur certains murs d'Athènes ou de Lisbonne aujourd'hui - c'est sans doute moins ceux-ci que l'univers de référence où s'inscrivent ces gestes qui leur donne sens.

Dans Koolkiller ou l'insurrection par les signes, un texte qu'il publie en i976, Jean Baudrillard analyse la signification de l'irruption des graffitis à New York au tout début des années 70 dans un contexte qui suit les grandes révoltes urbaines des années ig67-I97o. Dans la ville moderne, "chaque pratique, chaque instant de la vie quotidienne est assigné par de multiples codes à un espace/temps déterminé ${ }^{72}$ ». La ville historique, celle qui a vécu jusqu'au $\mathrm{xIx}^{\mathrm{e}}$ ou au début du $\mathrm{xx}^{\mathrm{e}}$ siècle, politico-industrielle et socialement hétérogène, cède rapidement la place à un environnement de signes qui la quadrillent de codes, et ce «sur la base d'une définition analytique: habitat, transport, travail, loisir, jeu, culture - autant de termes commutables» dans un espace à la fois indifférencié et hautement discriminant. Manière de venir rompre cet enfermement dans la "forme/signe», le tag vient subvertir l'ordre anonyme et référencé de la ville en requalifiant l'espace urbain en "territoire collectif par le biais d'appellations totémiques dissolvant la fonctionnalité de sa signalétique ${ }^{73}$ ». Signature illisible, le tag l'est volontairement, tout comme il est là pour recouvrir, dégrader, salir et perturber tous ces codes installés. Écriture sans souci de communiquer, signifiant sans signifié, il n'est pas cependant sans message, défi retissant un réseau sur le réseau, propre aux marges revendiquées d'une classe d'âge ou d'exclus, réaction "tribale» temporaire, d'individus ou de groupes en attente d'une hypothétique intégration dans le “réseau ${ }^{74}$ ». Cela n’a guère à voir avec nos illustres ancêtres qui

72 J. Baudrillard, L'échange symbolique et la mort, Paris, Gallimard, i976, p. II8-i28.

73 М. КокоRеFF, «Des graffitis dans la ville », Quaderni 6, Hiver 88/89, p. 85-86.

74 A. Guillain, “L'enfance du geste: écriture et graffiti », Communication et langages, $\mathrm{n}^{\circ}$ 97, $3^{\mathrm{e}}$ trimestre 1993, p. $46-48$. 
affichaient et leur nom et leurs exploits, comme ce Rennais qui se vantait d'avoir baisé vingt-cinq fois sa petite amie ${ }^{75}$.

Aucun des tagueurs actuels ne reproduit, même inconsciemment, un geste ancien, sauf à prendre uniquement en compte ce désir particulier d'expression. Les codes ont changé. Et cela même lorsque ces écritures urbaines revendiquent une esthétique. Le statut, qu'avec parfois la complicité des acteurs eux-mêmes les municipalités leur accordent en mettant à leur disposition des espaces à eux réservés, est souvent un moyen peu coûteux d'intégrer cette subversion au « réseau des réseaux» et de contrôler la prolifération de cette écriture sauvage. En ce sens, la référence au documentaire d'Agnès Varda est tout aussi décalée. L'espace propre à la peinture murale romaine est à ce point codifié qu'on aurait du mal à faire un parallèle entre les murs peints modernes et l'exceptionnelle représentation de bateau gravée à la pointe qui, comme s'il s'agissait d'un tableau, respecte parfaitement la structuration de la fresque monochrome dans une des pièces de la villa du Viély à Cucuron $^{76}$.

Si notre monde contemporain paraît sur ce point s'inscrire en rupture, il n'en a pas toujours été ainsi. Paradoxalement, le graffiti peut aussi traduire une certaine forme de réception de l'Antique, au moins de la Renaissance au XviI ${ }^{\mathrm{e}}$ voire au xIx siècle. Au terme d'un parcours d'un an passé à recueillir les graffitis apposés à Rome sur des œuvres majeures par une population de soldats, de touristes ou d'artistes, Charlotte Guichard propose d'y voir plus que la simple trace du passage de ces voyageurs, illustres ou anonymes ${ }^{77}$.

Julien Gracq parlait de "cette alluvion de mots qui recouvre Rome comme une palissade se recouvre d'affiches ${ }^{\mathbf{7 8}}$ ». Ce sont ici pour l'essentiel des noms propres, suivis d'une date, que l'on trouve gravés ou apposés à la frontière du visible. Manifestant par leur position sur le bord des œuvres un respect paradoxal pour celles-ci, ces signatures "enregistrent une expérience, celle d'avoir été au contact du monde enfoui de l'Antiquité79 ${ }^{\prime}$ ou des chefs-d'œuvre de prédécesseurs ou de contemporains illustres, Raphaël, les Carrache, etc. Elles sont comme autant de “reconnaissances de dettes», attestations d'une tradition commune qui lient entre eux voyageurs et artistes qui se succèdent et confessent ensemble les avoir soit copiés soit admirés, partageant ainsi les mêmes modèles d'exemplarité. C'est ainsi Johann Christian von Mannlich qui, en $\mathbf{1 7 6 7}$, grave son nom sur le stuc doré du soubassement de la galerie des Carrache, témoignant de ses journées passées à copier les fresques ${ }^{80}$ et dont le nom vient s'ajouter à une longue liste d'autres artistes qui ont fait de même ${ }^{81}$. C'est David, qui signe en 1784 , de manière presqu'invisible, sur une des fresques de la Farnésine, sous le nom de celui qui les restaura en i6g3 avec considération,

75 Les Murs murmurent, $\mathrm{n}^{\circ}$ 62, fig. I29, p. 148 sq.

76 Les Murs murmurent, fig. ı16, p. 132 ; n 55, fig. ı 3, p. 36.

77 Guichard, Graffitis, p. I5-3o.

78 J. GracQ, Autour des sept collines, Paris, Corti, ig88, p. 68.

79 Guichard, Graffitis, p. 52.

80 Guichard, Graffitis, p. I8.

81 Guichard, Graffitis, p. 75 sq. 
Carlo Maratta. Toutes ces marques tracent ainsi un double parcours, spatial et temporel, mais dont le but n'est pas de substituer un réseau tribal à un territoire fonctionnel commutable. À l'opposé, ces signes “actualisent les œuvres et les inscrivent dans un présent ${ }^{82}$ » aux temporalités multiples faites de raccourcis, d'oublis et de réminiscences. Qu'il s'agisse alors d'un artiste ou d'un simple amateur n'a guère d'importance: le témoignage d'émulation de l'artiste ou le simple acte de présence du touriste atteste d'un rapport quasi sacré à l'image qu'il n'est pas question ici de chercher à dégrader en y ajoutant un patronyme. Cette idée de dégradation ne vient que tardivement, portée par l'obsession moderne du patrimoine ${ }^{83}$ qui scelle ce que la réception pouvait avoir de vivant dans un "glacial enregistrement muséal ${ }^{84}$ ». Curieusement celui-ci semble contemporain de la transformation progressive de la ville en “polygone de signes ${ }^{85}$ » lorsque le graffiti est renvoyé à son unique dimension transgressive et provocatrice. Tout au plus ne reste-t-il aujourd'hui que le geste qui consiste à se “selfier» devant l'œuvre, ultime forme d'un hommage plus éminemment narcissique encore que ne pouvait l'être le graffiti.

Alors que ce graffiti moderne interroge l'histoire de l'art en donnant une dimension archéologique à l'histoire d'un chef-d'œuvre, parce qu'il parle d'historiographie et de réception autant, sinon plus, que de dégradations - et quand bien même, ces dernières font aussi partie de l'œuvre et de son histoire -, le graffiti antique paraît difficilement réductible à ses descendants modernes. Le “champ des signes », pour reprendre l'expression d'Alix Barbet, ne se déploie pas dans le même contexte. Objet d'histoire, le geste antique transmet un message univoque indépendamment d'une quelconque problématique de réception - tout au plus peut-il parfois s'agir de citations ou d'allusions à des textes littéraires contemporains $^{86}$. Malgré l'apparence et le contenu, identiques parfois, ou la forme des messages que laissent nos contemporains sur les murs des différents lieux de nos villes, il est malaisé de voir là l'héritage direct des témoignages de l'Antiquité, tant l'espace dans lequel ces graffs se déploient a changé depuis cent ans.

\author{
Manuel Royo \\ Université de Tours \\ 3, quai des Tanneurs \\ F-37 ooo Tours \\ CeTHiS EA 6298 \\ royo@univ-tours.fr
}

82 Guichard, Graffitis, p. I04sq.

83 Guichard, Graffitis, p. $\operatorname{Io} 8$ sq.; p. 135 sq.

84 Grace, Autour des sept collines, p. II7.

85 Baudrillard, Koolkiller, p. II8 sq.

86 P. Keegan, "Blogging Rome: Graffiti as a Speech-Act and Cultural Discourse», in Ancient Graffiti in Context: I65-ıgo; K. Milnor. Graffiti, chap. 4 (A Culture of Quotation: Virgil, Education, and Literary Ownership). 